\title{
Celebrating the 100th Anniversary of the Founding of the Communist Party of China: Successes and Challenges in Promoting the Nation's Health Through Sport and Physical Activity
}

\author{
Wei Gao and Keqiang Cao
}

The anniversary of the Communist Party of China (CPC), which was founded in July 1921, is around the corner. Many fields across the country are conducting reviews on the performance and achievements made by the CPC in the past 100 years. This is an important effort at this historic moment because these reviews will help facilitate the development of future plans, initiatives, and policies to continue improving the nation's economy, social wellbeing, and population health. In the field of sports science, the Journal of Sports \& Science (in Chinese) has recently published a series of articles, including the editorial "From the Sport for Save the Country and Strong the Country to Health China and Sports Power-A Sport Memorabilia Tour for the 100th Anniversary of the Founding of the Communist Party of China," that summarizes achievements made in sports. ${ }^{1}$

The article $^{1}$ highlights major milestones achieved under the leadership of the CPC in the fields of sport, exercise, and physical activity since 1921. It contains the leaders' words or research on sports in different eras, major laws or policies, major events including being the host for the Asian Games (1990, 2010, and 2022) and Olympic Games (2008 and 2022), guidelines on promoting sports for all, and implementation policies for the sports industry.

Clearly, under the leadership of the $\mathrm{CPC}$, the nation has made remarkable achievements in the field of sports. However, there remain some major challenges in meeting the nation's overall health goals. ${ }^{2}$ First, neither sport nor physical activities were given a prominent role in the Healthy China 2030. China's Central Party Committee and the State Council approved the Healthy China 2030 Planning Outline in 2016; however, most of the contents were about how to improve health by upgrading hospital treatment and management, universal health coverage, improvements in the supply of essential medicines, enhancement of health literacy, clinical care, and stronger disease prevention. Only one milestone relating to sport was mentioned: aiming to achieve 530 million people exercising regularly by 2030 . Second, there is a lack of quantity and access to sports venues in communities to allow broader promotion of physical activity among citizens of different age groups. Newly released national census data ${ }^{3}$ show that the China's total population has reached 1.41 billion and that $63.89 \%$ of them are urban citizens; the increase is having major ramifications on physical activity promotion and chronic disease prevention. There were approximately 12 sports venues for every 10,000 persons, or $1.46 \mathrm{~m}^{2}$ of activity space for every person. ${ }^{4}$ Therefore,

The authors are with the School of Economics and Management, Shanghai University of Sport, Shanghai, China. Cao (kqcao@sus.edu.cn) is corresponding author. increasing new sports facilities, opening more venues to the public, and making full use of venues are still great problems and challenges but very important issues, especially for medium and large cities. Third, the physical activity participation of children and adolescents still needs to be improved because there is generally a lack of attention to implementing evidence-based practice in communities. There has been a steady increase in body mass index for girls and boys during the economic expansion in recent years, so that overweight and obesity in youth are very common, resulting in poorer health-related quality of life..$^{5}$ Improving more than 170 million children's and adolescents' overall health has become a national priority. ${ }^{6}$

Over the past century, the CPC has gained considerable experience in solving practical problems. Several steps, however, can be taken at the policy level to increase sport, exercise, and physical activity participation among Chinese citizens. Policy has widely been regarded as an essential part of the solution to global physical inactivity. ${ }^{7}$ First, the government should transition the blueprint into action. There are a few areas that have made meeting nation's health promotion goal difficult, such as the top-down approach in policy implementation; lack of corporation and/or collaboration across disciplines; lack of practical, achievable milestones in health indicators; and lack of strong public health professions and infrastructures. Second, there are studies showing that sedentary behavior is associated with an increased risk of developing chronic disease and premature death among Chinese adults, especially for those with $\geq 10$ hours per day of sedentary time. ${ }^{8}$ Therefore, it is necessary to change the people's lifestyle, in schools for youth and in the community for adults, to increase moderate to vigorous physical activity levels and decrease sedentary time. Third, the government should provide more research funds in this field. Research findings of high scientific rigor would address problems and/or issues critical to public health, sports and exercise sciences, physical activity epidemiology, and clinical education and rehabilitation that have the potential to impact community and/or clinical practice.

It is hoped that the centenary anniversary will serve as a turning point from which the nation can draw important lessons from the past and strive forward to achieve the major health goals set by the CPC in increasing the population's level of physical activity and improving the health status and quality of life among Chinese citizens.

\section{Acknowledgments}

The authors would like to thank Fuzhong Li for his helpful comments on an early version of this paper. This work was supported by the Shanghai 
Philosophy and Social Science Planning Project (2019BTY004). Both authors declare that they have no competing interests.

\section{References}

1. The editorial office. From the sport for save the country and strong the country to health China and sports power-a sport memorabilia tour for the 100th anniversary of the founding of the Communist Party of China. [in Chinese].J Sports Sci. 2021;42:30-33.

2. Chen P, Li F, Harmer P. Healthy China 2030: moving from blueprint to action with a new focus on public health. Lancet Public Health. 2019;4: e447. PubMed ID: 31493840 doi:10.1016/S2468-2667(19)30160-4.

3. The CCTV. The data of the Seventh National Census released. http:// tv.cctv.com/2021/05/11/ARTIK6w5Q4Gnn7YKxZf9gZ2g210511. shtml. Accessed May 15, 2021.
4. Gao W, Cao K. Increasing public access to sports venues to promote physical activity among older adults in China. J Aging Phys Act. 2018;26:169-170. PubMed ID: 28952851

5. Bu T, Popovic S, Huang H, Fu T, Gardasevic J. Relationship between national economic development and body mass index in chinese children and adolescents aged 5-19 from 1986 to 2019. Front Pediatr. 2021;9:671504. PubMed ID: 33987156 doi:10.3389/fped.2021. 671504

6. Chen P, Wang D, Shen $\mathrm{H}$, et al. Physical activity and health in Chinese children and adolescents: expert consensus statement (2020). Br J Sports Med. 2020;54:1321-1331.

7. An R. Policy and physical activity. J Sport Health Sci. 2021;10:253254. PubMed ID: 33744227

8. Lin Y, Liu Q, Liu F, et al. Adverse associations of sedentary behavior with cancer incidence and all-cause mortality: a prospective cohort study. J Sport Health Sci. 2021. doi:10.1016/j.jshs.2021.04.002 